\title{
Isotope-selective photoionization for calcium ion trapping
}

\author{
D. M. Lucas, ${ }^{1}$ A. Ramos, ${ }^{1}$ J. P. Home, ${ }^{1}$ M. J. McDonnell, ${ }^{1}$ S. Nakayama, ${ }^{2}$ J.-P. Stacey, ${ }^{1}$ S. C. Webster, ${ }^{1}$ D. N. Stacey, \\ and A. M. Steane ${ }^{1}$ \\ ${ }^{1}$ Department of Physics, University of Oxford, Clarendon Laboratory, Parks Road, Oxford OX1 3PU, United Kingdom \\ ${ }^{2}$ Department of Information and Computer Science, Kagoshima University, 1-21-40 Korimoto, Kagoshima 890-0065, Japan
}

(Received 10 October 2003; published 27 January 2004)

\begin{abstract}
We present studies of resonance-enhanced photoionization for isotope-selective loading of $\mathrm{Ca}^{+}$into a Paul trap. The $4 s^{2}{ }^{1} S_{0} \leftrightarrow 4 s 4 p{ }^{1} P_{1}$ transition of neutral calcium is driven by a $423 \mathrm{~nm}$ laser and the atoms are photoionized by a second laser at $389 \mathrm{~nm}$. Isotope selectivity is achieved by using crossed atomic and laser beams to reduce the Doppler width significantly below the isotope shifts in the $423 \mathrm{~nm}$ transition. The loading rate of ions into the trap is studied under a range of experimental parameters for the abundant isotope ${ }^{40} \mathrm{Ca}^{+}$. Using the fluorescence of the atomic beam at $423 \mathrm{~nm}$ as a measure of the Ca number density, we estimate a lower limit for the absolute photoionization cross section of 170(60) Mb. We achieve loading and laser cooling of all the naturally occurring isotopes, without the need for enriched sources. Laser heating/cooling is observed to enhance the isotope selectivity. In the case of the rare species ${ }^{43} \mathrm{Ca}^{+}$and ${ }^{46} \mathrm{Ca}^{+}$, which have not previously been laser cooled, the loading is not fully isotope selective, but we show that pure crystals of ${ }^{43} \mathrm{Ca}^{+}$may nevertheless be obtained. We find that for loading ${ }^{40} \mathrm{Ca}^{+}$the $389 \mathrm{~nm}$ laser may be replaced by an incoherent source.
\end{abstract}

DOI: $10.1103 /$ PhysRevA.69.012711

PACS number(s): 32.80.Fb, 32.80.Rm, 32.80.Pj

\section{INTRODUCTION}

Resonance-enhanced photoionization for loading ion traps was first demonstrated with magnesium and calcium ions by Kjaergaard et al. [1]. The same group has recently used crossed-beam Doppler-free excitation of the $272 \mathrm{~nm}$ $4 s^{2}{ }^{1} S_{0} \leftrightarrow 4 s 5 p{ }^{1} P_{1}$ calcium transition, followed by photoionization, to load selectively all the naturally occurring isotopes of this element; the loading was not observed directly, but by using charge exchange to infer the presence of the other isotopes from the fluorescence of laser-cooled ${ }^{40} \mathrm{Ca}^{+}$ ions which replaced them [2]. Gulde et al. showed that, using the $4 s^{2}{ }^{1} S_{0} \leftrightarrow 4 s 4 p{ }^{1} P_{1}$ Ca transition at $423 \mathrm{~nm}$, followed by excitation close to the continuum by an ultraviolet photon, photoionization is around five orders of magnitude more efficient than conventional electron bombardment ionization [3].

As pointed out by these authors, photoionization has a number of advantages over electron bombardment. Only the desired species is loaded into the ion trap, allowing pure crystals of particular isotopes to be obtained. Since no electron beam is involved, there is no charging of insulating parts of the trap structure, which leads to drifting electric fields that for many experiments must be accurately compensated. The efficiency of the photoionization process allows much lower number densities of neutral atoms in the interaction region, greatly reducing the quantities of material sputtered onto the trap electrodes; it has been shown that clean electrode surfaces reduce the heating rate of trapped ions from the motional ground state [4], an important consideration for quantum logic experiments in ion traps. For experiments in which high-finesse optical cavities are combined with ion traps, the lower atomic beam density reduces the degradation of the mirror surfaces and can avoid the need for a separate "loading trap" $[5,6]$.

Our particular interest lies in using ${ }^{43} \mathrm{Ca}^{+}$as an ion qubit in quantum logic experiments [7]. This ion has a number of advantages as a qubit [8,9]; it is also an attractive candidate for a trapped ion optical frequency standard $[10,11]$. The most obvious difficulty in working with ${ }^{43} \mathrm{Ca}^{+}$is its low natural abundance of $0.135 \%$. In this paper we describe the development of a photoionization system capable of loading ${ }^{43} \mathrm{Ca}^{+}$into a Paul trap from a natural abundance source. (Isotopically enriched sources are available, although the maximum enrichment of $\sim 80 \%$ [12] means that it would still be advantageous to use an isotope-selective method for reliable loading of this isotope.)

We describe the crossed atomic and laser beam setup for excitation of the $423 \mathrm{~nm} 4 s^{2}{ }^{1} S_{0} \leftrightarrow 4 s 4 p{ }^{1} P_{1}$ transition with a Doppler width narrow compared with the isotope shifts. Spectroscopy of the atomic beam is used to ensure that the intensity of the $423 \mathrm{~nm}$ laser is below saturation (to avoid reducing the isotope selectivity of this step by power broadening), and also to estimate the number density of atoms in the beam. Photoionization from the $4 s 4 p{ }^{1} P_{1}$ level is achieved by a $389 \mathrm{~nm}$ photon; since we expect the photoionization cross section to be only weakly dependent on the photon energy, we use a nonstabilized diode laser for this step. We study the photoionization trap loading rate as a function of the power and detuning of the $423 \mathrm{~nm}$ laser, and as a function of the power of the $389 \mathrm{~nm}$ laser. We find that the $389 \mathrm{~nm}$ laser photoionizes equivalently above or below lasing threshold, and that it can be replaced by a much cheaper incoherent source if high photoionization rates are not required. We estimate the absolute photoionization loading efficiency and compare it with that for electron bombardment ionization in our apparatus.

The sensitivity of the loading rate as a function of the 423 $\mathrm{nm}$ detuning determines the isotope selectivity which can be attained. However, for very rare species with mass number $X$, a practical limitation is the rate at which charge exchange replaces the desired, trapped, species ${ }^{X} \mathrm{Ca}^{+}$by the abundant 

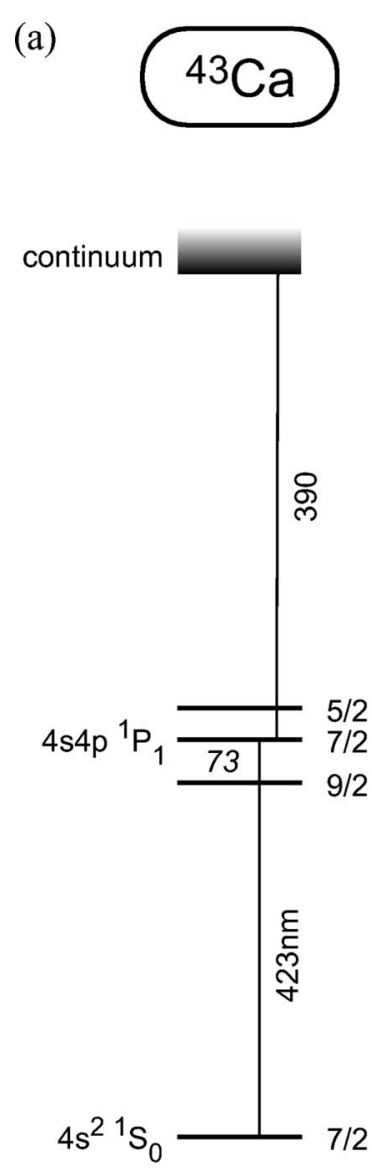

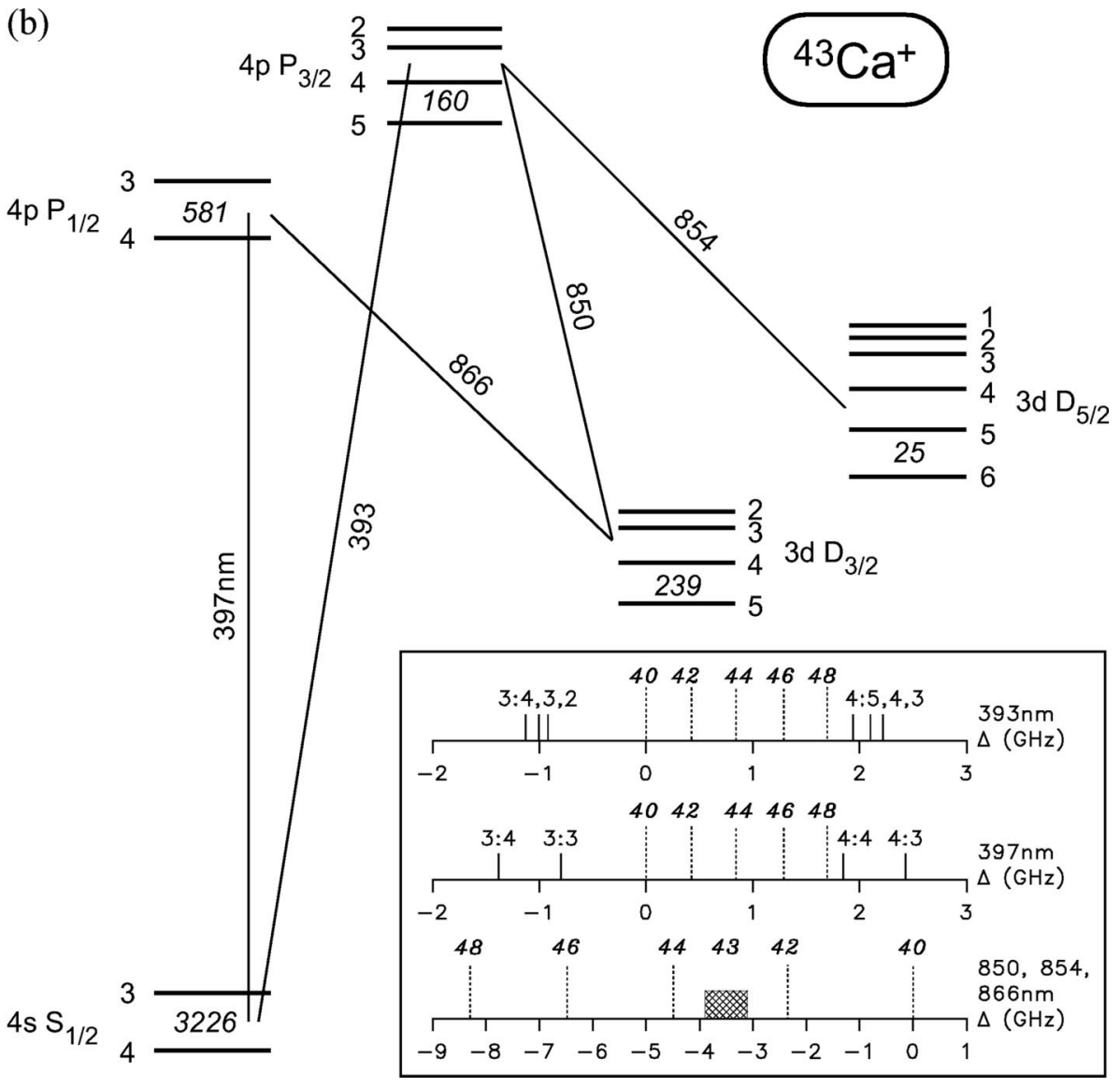

FIG. 1. Energy levels of interest in ${ }^{43} \mathrm{Ca}$ (for photoionization) and ${ }^{43} \mathrm{Ca}^{+}$(for laser cooling), with hyperfine levels labeled by the total angular momentum $F$. The nuclear spin is $I=7 / 2$. The largest hyperfine splitting in each term is indicated in megahertz; the levels approximately conform to an interval rule $[15,16]$. Diagrams for the even isotopes are the same but with no hyperfine structure. (a) ${ }^{43} \mathrm{Ca}$ : the lifetime of the $4 s 4 p{ }^{1} P_{1}$ level is 4.49(4) ns [17] and the natural linewidth of the $423 \mathrm{~nm}$ transition 35.4(3) MHz. Photoionization from the $4 s 4 p{ }^{1} P_{1}$ level requires a photon of wavelength less than $389.8 \mathrm{~nm}$. (b) ${ }^{43} \mathrm{Ca}^{+}$: the natural widths of the ultraviolet transitions are approximately $23 \mathrm{MHz}$, the lifetimes of the metastable $D$ levels about 1.2s [18]. Inset: Positions of the hyperfine components of the $393 \mathrm{~nm}$ and $397 \mathrm{~nm}$ transitions (solid lines, labeled with $F_{\text {lower }}: F_{\text {upper }}$ ) and the isotope shifts of the even isotopes (dotted lines, labeled with the mass number), as a function of detuning $\Delta$ relative to ${ }^{40} \mathrm{Ca}^{+}$. The isotope shifts in the infrared transitions (similar in all three transitions) are also shown, without the hyperfine details for ${ }^{43} \mathrm{Ca}^{+}$; note the change of scale.

${ }^{40} \mathrm{Ca}$ issuing from the oven. We demonstrate loading of all the naturally occurring isotopes, including ${ }^{43} \mathrm{Ca}^{+}$and the $0.004 \%$ abundant ${ }^{46} \mathrm{Ca}^{+}$. The limited isotope selectivity attainable for these two rare species means that some form of "purification" of the ions loaded is necessary $[13,14]$; in the case of ${ }^{43} \mathrm{Ca}^{+}$we show that this is possible with no loss of the desired species. Hence it should be possible to load arbitrarily large crystals of this important ion.

Level diagrams for ${ }^{43} \mathrm{Ca}$ and ${ }^{43} \mathrm{Ca}^{+}$, showing the energy levels and transitions relevant to these experiments, are shown in Fig. 1. Isotope shifts in the six transitions shown are summarized in Table I, together with the abundances of the six naturally occurring isotopes.

\section{APPARATUS}

\section{A. Ion trap}

The apparatus consists of a linear radio-frequency (rf, 6.2 $\mathrm{MHz}$ ) Paul trap in an ultrahigh-vacuum system (background pressure $<2 \times 10^{-11}$ torr), with diode laser systems available to drive all the transitions shown in Fig. 1. Details of the trap are given in [18]. Typical axial and radial frequencies used in the present experiments are $170 \mathrm{kHz}$ and $500 \mathrm{kHz}$, respectively. Auxiliary dc electrodes are used to compensate stray electric fields to the level of about $1 \mathrm{~V} / \mathrm{m}$ using a photon-rf correlation technique applied to the fluorescence from a single trapped ion [21]. A magnetic field of $\sim 3 \mathrm{G}$ is applied to prevent optical pumping into magnetic substates [11].

\section{B. Lasers}

The lasers are all of the external-cavity grating-stabilized Littrow design, except the $389 \mathrm{~nm}$ laser which has no grating stabilization. The blue and ultraviolet laser diodes are all $\mathrm{GaN}$ devices, the infrared ones GaAlAs. The gratingstabilized lasers may be locked to tunable, stabilized, optical cavities to reduce linewidths and medium-term frequency drift below $5 \mathrm{MHz}$. The laser frequencies are set within about $100 \mathrm{MHz}$ of the ionic transitions with wave meters. 
TABLE I. Abundances of the naturally occurring calcium isotopes [19], and isotope shifts for Ca and $\mathrm{Ca}^{+}$ transitions relevant to this work. Shifts in the $423 \mathrm{~nm}$ transition are taken from [16]; uncertainties quoted are below $1 \mathrm{MHz}$. Shifts in the ultraviolet $\mathrm{Ca}^{+}$transitions were measured in [20]; we quote the (more accurate) measurements for the $397 \mathrm{~nm}$ transition: the shifts at $393 \mathrm{~nm}$ are all consistent with these. Shifts in the infrared transitions are from [15]; the data given are for the $854 \mathrm{~nm}$ transition, which are again consistent with those for the other two lines. For the odd isotope, the shift of the center of gravity (c.g.) of the hyperfine components of each transition is given; note that in the $\mathrm{Ca}^{+} S-P$ lines, the hyperfine structure is significantly larger than the isotope shift, Fig. 1(b).

\begin{tabular}{lcccc}
\hline \hline & & \multicolumn{3}{c}{ Isotope shifts (MHz) } \\
\cline { 3 - 5 } $\begin{array}{c}\text { Mass } \\
\text { number }\end{array}$ & $\begin{array}{c}\text { Natural } \\
\text { abundance }\end{array}$ & $\begin{array}{c}\mathrm{Ca}^{1} S_{0^{-}}{ }^{1} P_{1} \\
423 \mathrm{~nm}\end{array}$ & $\begin{array}{c}\mathrm{Ca}^{+} S-P \\
397 \mathrm{~nm}[393 \mathrm{~nm}]\end{array}$ & $854 \mathrm{~nm}[850,866 \mathrm{~nm}]$ \\
\hline 40 & $96.9 \%$ & 0 & 0 & 0 \\
42 & $0.647 \%$ & 394 & $425(6)$ & $-2350(4)$ \\
43 (c.g.) & $0.135 \%$ & 612 & $688(17)$ & $-3465(4)$ \\
44 & $2.09 \%$ & 774 & $842(3)$ & $-4495(4)$ \\
46 & $0.004 \%$ & 1160 & $1287(4)$ & $-6478(8)$ \\
48 & $0.187 \%$ & 1513 & $1696(6)$ & $-8288(7)$ \\
\hline \hline
\end{tabular}

Doppler-free saturated absorption spectroscopy in a calcium hollow cathode is used to monitor the $423 \mathrm{~nm}$ laser frequency relative to the $4 s^{2}{ }^{1} S_{0} \leftrightarrow 4 s 4 p{ }^{1} P_{1}$ atomic transition. The vacuum wavelength of the $389 \mathrm{~nm}$ free-running laser was measured using a grating spectrograph to be 388.9(1) $\mathrm{nm}$ at $20^{\circ} \mathrm{C}$ (with negligible dependence on drive current); above lasing threshold, the spectral width is expected to be $\$ 100 \mathrm{MHz}$, below threshold $\sim 3 \mathrm{~nm}$. For convenience of alignment, the $423 \mathrm{~nm}$ and $389 \mathrm{~nm}$ laser beams are both injected into the same single-mode optical fiber to transport them to the trap; this ensures that the beams are well overlapped in the interaction region, which is centered on the trap. The transmission of the fiber is $\sim 30 \%$ at $423 \mathrm{~nm}$ and $\sim 10 \%$ at $389 \mathrm{~nm}$. The maximum intensities at the center of these beams are $\sim 50 \mathrm{~mW} / \mathrm{mm}^{2}$ at $423 \mathrm{~nm}$ and $\sim 5 \mathrm{~mW} / \mathrm{mm}^{2}$ at $389 \mathrm{~nm}$, and they are linearly polarized at right angles to each other. Typical intensities of the cooling lasers at the trap are $10 \mathrm{~mW} / \mathrm{mm}^{2}$ at $397 \mathrm{~nm} ; 20 \mathrm{~mW} / \mathrm{mm}^{2}$ at $393 \mathrm{~nm} ; 6$ $\mathrm{mW} / \mathrm{mm}^{2}$ at $866 \mathrm{~nm} ; 200 \mathrm{~mW} / \mathrm{mm}^{2}$ at $854 \mathrm{~nm} ; 650$ $\mathrm{mW} / \mathrm{mm}^{2}$ at $850 \mathrm{~nm}$. These intensities are based on the measured spot sizes for the various beams, which range from 40 to $300 \mu \mathrm{m}$.

\section{Detection system}

Fluorescence from the interaction region at $423 \mathrm{~nm}$ and $397 \mathrm{~nm}$ acts as a diagnostic of neutral calcium and calcium ions, respectively. The trap region is imaged by a compound lens onto an aperture to reject scattered light; further lenses reimage the trap, via a violet filter, onto a photomultiplier (PMT). A movable beam splitter allows a portion of the light to be directed to a charge-coupled device (CCD) camera; the camera is used to detect the presence of nonfluorescing ions in the trap (revealed as "gaps" in an otherwise regular crystal), and to check whether the ions form a cold crystal or a hot cloud. The net detection efficiency of the PMT system, including the solid angle subtended by the lens and the quantum efficiency of the PMT, was measured to be $0.13(2) \%$ at $397 \mathrm{~nm}$; the photon count rate observed from a single, cold,
${ }^{40} \mathrm{Ca}^{+}$ion under conditions of near saturation is consistent with this figure. The net detection efficiency at $423 \mathrm{~nm}$ is $\eta=0.15(3) \%$.

The discriminated pulses from the PMT are counted directly by $10 \mathrm{MHz}$ on-board counters in the computer which controls the experiment; two alternately gated counters are used to eliminate read-out delay. For the large peak photon counting rates encountered in studies of the $423 \mathrm{~nm}$ fluorescence (up to $\sim 10^{6} \mathrm{~s}^{-1}$ ), we correct the measured PMT count rate $S^{\prime}$ for the maximum counting rate $S_{\max }$ $=10 \mathrm{MHz}$, assuming $S \ll S_{\max }$, to obtain the true count rate $S \approx S^{\prime} /\left(1-S^{\prime} / S_{\max }\right)$.

\section{Calcium beam}

The calcium source consists of an oven made from stainless steel tube (diameter $2 \mathrm{~mm}$, wall thickness $0.1 \mathrm{~mm}$ ) filled with granules of calcium metal, closed by crimping at each end, and with an orifice (estimated area $\sim 1 \mathrm{~mm}^{2}$ ) filed in the side facing toward the trap. The oven is heated by passing a current (between $3 \mathrm{~A}$ and $6 \mathrm{~A}$ ) along its length, which produces a beam of calcium atoms directed toward the trap. Estimated oven temperatures are in the range 500-650 K, depending on the current used (Sec. III C).

The oven is situated 22(2) $\mathrm{mm}$ from the center of the trap. The atomic beam is collimated by the rf trap electrodes to an effective aperture of length $l=1.7(1) \mathrm{mm}$ along the direction of the $423 \mathrm{~nm} / 389 \mathrm{~nm}$ laser beams. However, the detection system images only a limited length of the interaction region (inset of Fig. 2), so the effective aperture for observation of the $423 \mathrm{~nm}$ fluorescence is reduced to $l^{\prime}$ $=0.44(4) \mathrm{mm}$. Since the oven orifice is of comparable dimensions to $l, l^{\prime}$, the angular divergence of the beam is somewhat larger than these distances would otherwise imply.

\section{SPECTROSCOPY OF THE ATOMIC BEAM}

We describe in this section spectroscopy of the $423 \mathrm{~nm}$ transition, using the calcium atomic beam from the oven. For 

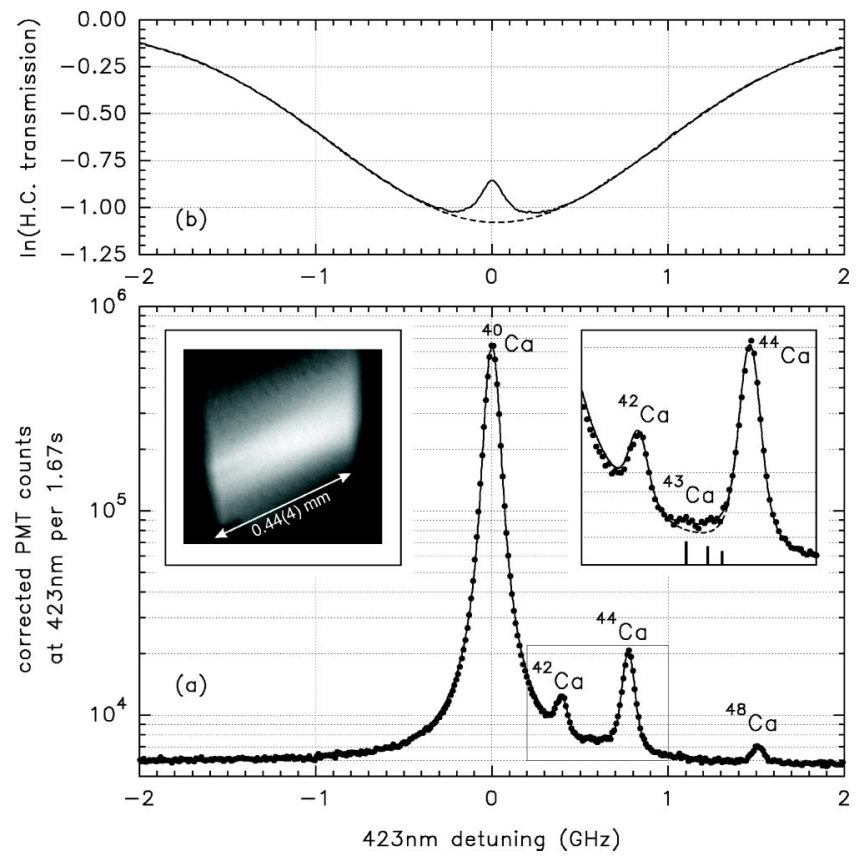

FIG. 2. (a) Fluorescence spectrum from the atomic beam at an oven current of $5.0 \mathrm{~A}$ and a laser power below saturation, $P_{423}$ $\sim 6 \mu \mathrm{W}$. The laser was locked to a tunable, stabilized, optical cavity to minimize drift during the scan; the frequency scale was determined using a second, confocal, cavity $(600 \mathrm{MHz}$ free spectral range). The best-fit Voigt profile is shown (solid line), where the amplitude, linear baseline, frequency offset, and Lorentzian and Gaussian widths were all floated simultaneously. The total FWHM is $76 \mathrm{MHz}$. The abundances, isotope shifts and hyperfine splittings were given the known values [16,19]. Right inset: Magnification of the region containing the three hyperfine components of ${ }^{43} \mathrm{Ca}$, whose expected positions are indicated. The dashed line shows the best fit when the ${ }^{43} \mathrm{Ca}$ components are omitted from the theoretical profile. The largest contribution to the signal by ${ }^{43} \mathrm{Ca}$ is about $22 \%$ of the total signal above background. Left inset: Fluorescence at ${ }^{40} \mathrm{Ca}$ line center from the interaction region, as observed on the CCD camera. The imaging system aperture limits the length of the $423 \mathrm{~nm}$ beam observed to 0.44(4) mm. (b) Simultaneous Dopplerfree saturated absorption signal from the hollow cathode lamp; logarithmic transmission is shown. The Doppler-free peak (FWHM $\sim 170 \mathrm{MHz}$ ) is superimposed on the Doppler-broadened background (FWHM $\sim 2.2 \mathrm{GHz}$, dashed curve), and is centered within $10 \mathrm{MHz}$ of the ${ }^{40} \mathrm{Ca}$ fluorescence peak. Peaks due to the other isotopes are too small to be visible.

optimal isotope selectivity in the photoionization loading, the narrowest possible linewidth of this transition is required. Ideally the linewidth should be small compared with the isotope shifts (Table I), with the lower limit being set by the 35.4(3) MHz natural width. Other contributions to the homogeneous width include power broadening (saturation), transit-time broadening, and laser linewidth. Collisional broadening is entirely negligible at the low number densities involved here. The inhomogeneous broadening (Doppler width) is determined by the oven temperature, the collimation angle of the atomic beam, and the angle between the laser beam and the atomic beam.

For orthogonal beams, the velocity distribution approxi- mates to a Gaussian whose width compared with that in the oven is determined by the collimation geometry and the oven orifice ([22], Sec. 10.1). For nonorthogonal beams, the velocity distribution broadens and becomes asymmetric, resulting in a broadening and shift when it is convolved with the homogeneous profile.

\section{A. Alignment of the crossed beams}

To ensure orthogonal alignment of the laser and atomic beams, we minimize the width of the $423 \mathrm{~nm}$ fluorescence spectrum as a function of the angle between the beams. The power of the $423 \mathrm{~nm}$ laser $P_{423}$ is set safely below the saturation level for maximum sensitivity (see next section). A fluorescence spectrum with the alignment optimized is shown in Fig. 2, together with a simultaneously acquired Doppler-free hollow cathode spectrum. From several such spectra we find that the offset between the ${ }^{40} \mathrm{Ca}$ fluorescence peak and the hollow cathode saturated-absorption peak is at most $10 \mathrm{MHz}$ (after accounting for a pressure redshift of up to $13 \mathrm{MHz}$ in the hollow cathode lamp due to 6 torr of neon buffer gas [23]). This indicates a beam misalignment of at most $\sim 12$ mrad.

The fitted Voigt profile shown has Lorentzian full width at half maximum (FWHM) $39 \mathrm{MHz}$ and Gaussian FWHM 52 MHz. The transit-time broadening is estimated from the measured laser spot size and the approximate atomic velocity (Sec. III C) to be 2(1) MHz. The laser linewidth estimated from the error signal when the laser is locked to a stabilized reference cavity is 4(1) MHz. The Gaussian width is consistent with the collimation aperture $l^{\prime}=0.44(4) \mathrm{mm}$ and the estimated oven temperature $T \sim 610 \mathrm{~K}$ if we model the oven as an extended source of size $\sim 1 \mathrm{~mm}$.

\section{B. Saturation broadening}

It is important to avoid saturation broadening of the 423 $\mathrm{nm}$ transition. Since it is difficult to estimate absolute intensities accurately, and the atomic response is averaged over the laser beam profile, we studied the saturation of the transition empirically, by varying the laser power. Results, obtained after alignment of the crossed beams, are shown in Fig. 3. Saturation effects begin, in both the peak fluorescence and the Lorentzian linewidth, above a laser power of about $10 \mu \mathrm{W}$.

The photoionization rate will increase as the population of the $4 s 4 p{ }^{1} P_{1}$ state, i.e., linearly until saturation sets in. Since the atomic beam is much larger than the laser beam, however, the total number of atoms in the upper state will continue to increase even well above saturation, although more slowly with increasing power. The isotope selectivity will decrease roughly as the square of the Lorentzian linewidth, for fixed Gaussian width (the wings of the Voigt profile are determined overwhelmingly by the Lorentzian component, for the Gaussian width involved here). To maximize the photoionization rate without sacrificing isotope selectivity, we work at powers in the range $5-15 \mu \mathrm{W}$ for photoionization loading.

Specifically, the fractional population of the $4 s 4 p{ }^{1} P_{1}$ $M_{J}=0$ upper state as a function of detuning $\Delta_{423}$ at $\lambda$ 

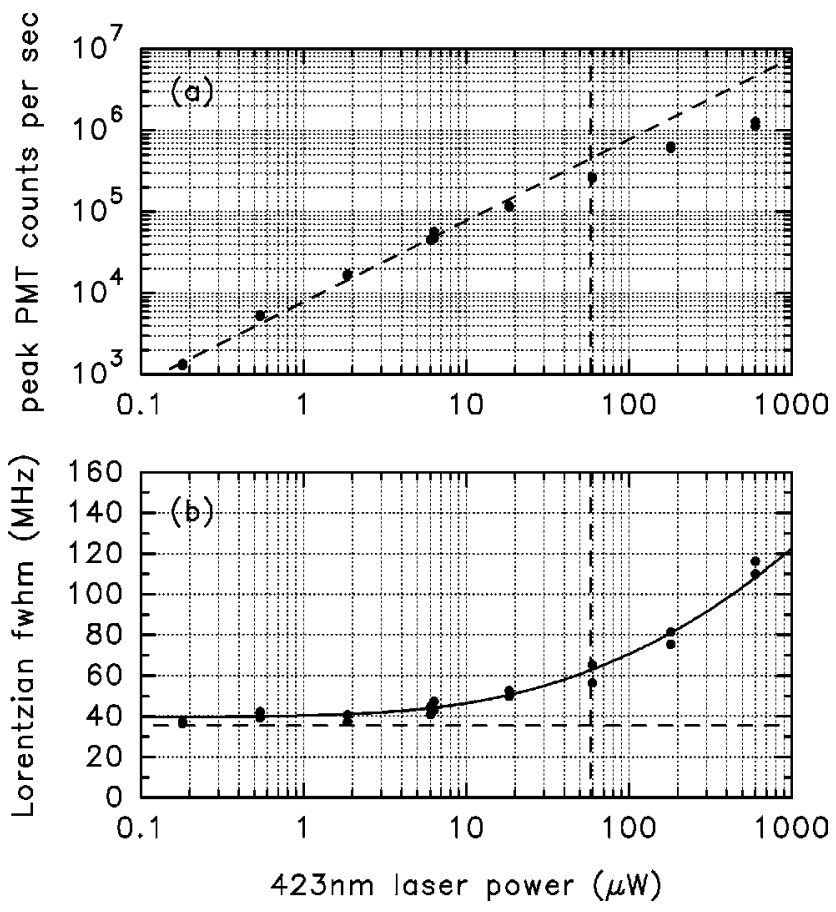

FIG. 3. Fluorescence and Lorentzian linewidth on the $423 \mathrm{~nm}$ transition, as a function of laser power $P_{423}$, obtained from fitting $423 \mathrm{~nm}$ spectra with Voigt profiles (whose Gaussian width was fixed at $51 \mathrm{MHz}$ ). The oven current was $4.25 \mathrm{~A}$. The vertical dashed lines indicate the power at which the intensity at the center of a beam with $100 \mu \mathrm{m}$ spot size reaches $I_{\text {sat }}=2 \pi h c A / \lambda^{3}$ $=3.68 \mathrm{~mW} / \mathrm{mm}^{2}$. (a) Peak (corrected) PMT count rate above background on the ${ }^{40} \mathrm{Ca}$ component. A straight line $(y \propto x)$ is fitted to the data for $P_{423}<10 \mu \mathrm{W}$; the onset of saturation is visible as the signal drops below this line at high powers. (b) Analyzed Lorentzian FWHM for the same data. The linewidth starts to increase for power above about $10 \mu \mathrm{W}$. The horizontal dashed line indicates the $35 \mathrm{MHz}$ natural linewidth. The curve shows the expected homogeneous FWHM, obtained by averaging the atomic response over a circular Gaussian beam with $100 \mu \mathrm{m}$ spot size and assuming a 4 $\mathrm{MHz}$ laser linewidth.

$=423 \mathrm{~nm}$ is, for linearly polarized laser light of intensity $I$,

$$
n_{4 s 4 p}\left(\Delta_{423}\right)=\frac{\frac{3}{2} \operatorname{IA} \beta}{\left(2 \pi \Delta_{423}\right)^{2}+\beta^{2}+3 I A \beta},
$$

where $A$ is the Einstein coefficient for the transition ( $A$ $=2.23 \times 10^{8} \mathrm{~s}^{-1}$; the weak decay route to $4 s 3 d^{1} D_{2}$ may be neglected since the calculated lifetime against this decay [17] is long compared to the transit time across the laser beam), $\beta=\left(2 \pi \Gamma_{\mathrm{L}}+A\right) / 2$ with $\Gamma_{\mathrm{L}}$ the laser linewidth, the intensity is measured in units of a saturation intensity $I_{\text {sat }}$ $=2 \pi h c A / \lambda^{3}$, and $\Delta_{423}, \Gamma_{\mathrm{L}}$ are in units of Hz. This expression must be integrated over the spatial profile of the laser beam to obtain the expected signal from the atomic beam; the resulting homogeneous FWHM, assuming a circular Gaussian beam and a laser linewidth $\Gamma_{\mathrm{L}}=4 \mathrm{MHz}$, is plotted in Fig. 3(b).
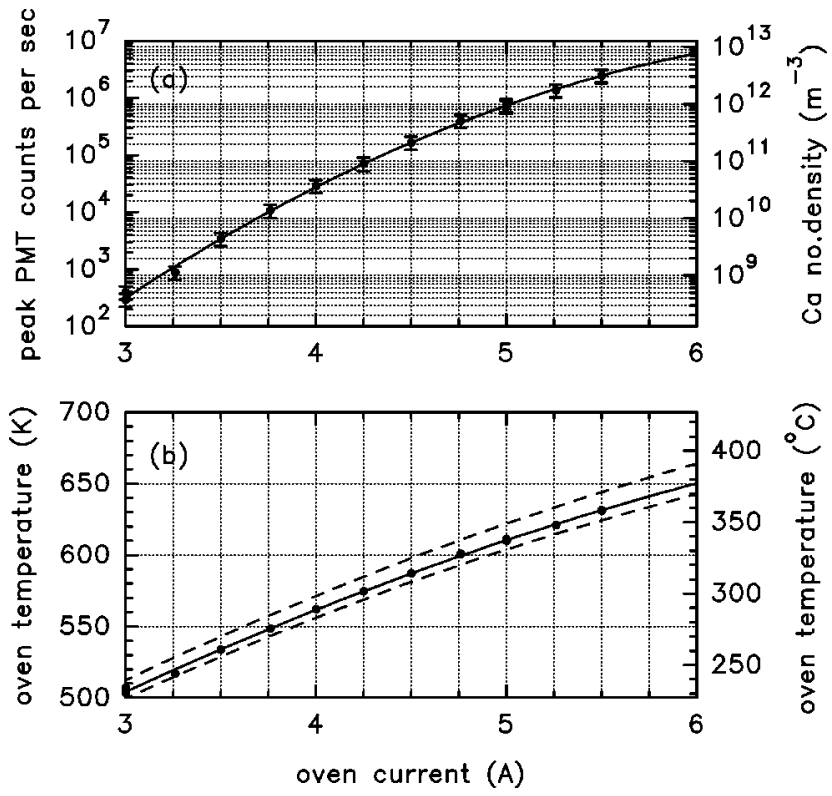

FIG. 4. Calcium number density and oven temperature as a function of oven current. (a) Corrected photon count rate above background on the ${ }^{40} \mathrm{Ca}$ peak obtained from fitting $423 \mathrm{~nm}$ spectra with Voigt profiles, where the Lorentzian width was fixed at 41 $\mathrm{MHz}$ from a spectrum at $4.25 \mathrm{~A}$ oven current. The right-hand ordinate gives the calcium number density in the interaction region deduced from this fluorescence rate as described in the text, and the error bars refer to the number density. A quadratic regression fit is shown (solid line). At $3 \mathrm{~A}$, the signal was so small that the line shape was poorly determined and the Gaussian width was also fixed for the fit. (b) Estimated oven temperature based on the number density in (a), with quadratic regression (solid line). The dashed lines indicate the temperature range implied by the $50 \%$ uncertainty assigned to the oven orifice area $\sigma=1.0(5) \mathrm{mm}^{2}$.

\section{Number density and temperature in the atomic beam}

We can use the peak ${ }^{40} \mathrm{Ca}$ fluorescence at $423 \mathrm{~nm}$ to deduce the number density of calcium atoms in the atomic beam, using the known line strength and the measured photon collection efficiency. This in turn permits an estimate of the absolute photoionization loading efficiency. By measuring the number density as a function of oven current we can also compare the efficiency of photoionization trap loading with the electron bombardment method (which have similar loading rates at very different oven currents).

The peak fluorescence as a function of oven current is shown in Fig. 4(a). The laser power was set below saturation, and under these conditions the peak corrected photon count rate $S$ is related to the number density $N$ of atoms in the ground state by

$$
S=\frac{3 \lambda^{3} A}{8 \pi h c} P_{423} l^{\prime} \eta r \mathcal{V}(0) a_{40} N,
$$

where $a_{40}=96.9 \%$ is the ${ }^{40} \mathrm{Ca}$ abundance, $l^{\prime}=0.44(4) \mathrm{mm}$ the length of the interaction region observed through the imaging aperture (measured along the laser beam direction), $\eta=0.15(3) \%$ the net collection efficiency of the detection system at $\lambda=423 \mathrm{~nm}, r=1.43(2)$ a geometrical factor aris- 
ing from the angular distribution of the fluorescence, and $\mathcal{V}(0)$ the value of the fitted Voigt profile at line center (which is normalized such that $\left.\int_{0}^{\infty} \mathcal{V}\left(\nu-\nu_{0}\right) d \nu=1\right)$. The fitted values of $\mathcal{V}(0)$ show a slight decrease with increasing oven current, due to increasing inhomogeneous width as the temperature rises, but this is no greater than the $\sim 10 \%$ fitting uncertainty, and for the purpose of Fig. 4(a) we use the average value $\overline{\mathcal{V}(0)}=9.9(1.0) \mathrm{GHz}^{-1}$ so that the number density $N\left(\mathrm{~m}^{-3}\right)=1.3(3) \times 10^{6} \mathrm{~S}\left(\mathrm{~s}^{-1}\right)$ and it can be plotted on the same ordinate.

For a small interaction region on the axis of the atomic beam, the number density $N$ in the interaction region is related to that in the oven, $N_{0}$, by

$$
N=\frac{N_{0} \sigma}{4 \pi d^{2}}
$$

where $\sigma$ is the area of the oven orifice and $d$ the distance from the orifice to the interaction region [[24], Sec. 4.2]. In our apparatus, $d=22(2) \mathrm{mm}$ but $\sigma$ is not accurately known. However, since number density depends strongly on temperature $T$, we can make a reasonable estimate of $T$ by assuming that $\sigma=1.0(5) \mathrm{mm}^{2}$, the value suggested by our profile analysis. We then find a temperature that satisfies $p$ $=N_{0} k T$ and the known vapor pressure curve $p=p(T)$ simultaneously [25]. This is shown in Fig. 4(b) and ranges between about $500 \mathrm{~K}$ and $650 \mathrm{~K}$. The temperature is useful for estimating the mean velocity along the beam $\bar{v}=\sqrt{\pi k T / 2 M}$ for atoms of mass $M: \bar{v}=433(4) \mathrm{m} / \mathrm{s}$ for ${ }^{40} \mathrm{Ca}$ at $T$ $=575(10) \mathrm{K}$.

\section{Conclusion}

With the $423 \mathrm{~nm}$ beam well aligned orthogonal to the atomic beam, and the laser power set below saturation, we observe a linewidth of $76 \mathrm{MHz}$ (FWHM), made up of Lorentzian and Gaussian contributions of about $39 \mathrm{MHz}$ and $52 \mathrm{MHz}$, respectively. This compares favorably with the isotope shifts, Table I. We do not use these data to predict relative loading probabilities for the different isotopes, however, since the collimation angle of the atomic beam for loading the trap is greater than that relevant to the $423 \mathrm{~nm}$ fluorescence $\left(l>l^{\prime}\right)$ and the Gaussian contribution to the linewidth will be correspondingly larger. A quantitative estimate of this is difficult since the angular divergence depends on the sizes of the oven orifice and the trap capture region, neither of which is accurately known. Instead, we investigate the actual photoionization loading rate as a function of $423 \mathrm{~nm}$ detuning, as described in the following section.

\section{PHOTOIONIZATION LOADING STUDIES}

\section{A. Expected form of photoionization cross section}

Calculations [26] of the cross section $Q$ for photoionization from the $4 s 4 p$ state suggest that, at $389 \mathrm{~nm}, Q$ lies in the range $60-280 \mathrm{Mb}\left(1 \mathrm{Mb}=10^{-22} \mathrm{~m}^{2}\right)$. The large range results from the existence of an autoionizing resonance close to the ionization threshold, whose wavelength is uncertain to $\sim 5 \mathrm{~nm}$. Since the energy of the resonance is not well known, we do not attempt to choose a particular laser wavelength, beyond ensuring that it is below the threshold required for ionization. The width of the resonance is certainly large compared to the laser linewidth, so there is no need for the laser to be carefully frequency stabilized.

The electric fields present in a Paul trap can allow ionization to occur even for excitation slightly below the continuum limit [3]. For the typical velocity estimated above, atoms cross the photoionization laser beams in $\sim 0.2 \mu \mathrm{s}$ and all atoms are thus able to sample the extrema of the rf field (period $0.16 \mu \mathrm{s}$ ). The peak field varies across the trap, but is at most $\sim 10^{5} \mathrm{~V} / \mathrm{m}$. A rough estimate [[27], Sec. III.A.4] of the effect of such a field on the ionization threshold may be made by assuming a hydrogenic potential $V(x)=$ $-e / 4 \pi \epsilon_{0} x$ near the continuum and equating this to the potential $(-e E x)$ of the electron in the external field $E$; the result is that the ionization limit could be shifted at the furthest to a wavelength of $391.3 \mathrm{~nm}$. However, no significant enhancement of the photoionization cross section is expected by using wavelengths closer to this shifted ionization limit, even for monochromatic light, because the oscillatory electric field blurs out the discrete energy levels of the atom near the continuum and the average transition probability is no higher than that within the continuum [[28], Sec. 18.6].

\section{B. Photoionization loading rate measurements}

A typical photoionization loading curve is shown in Fig. 5 , where the fluorescence at $397 \mathrm{~nm}$ from cold ${ }^{40} \mathrm{Ca}^{+}$ions in the trap is plotted as a function of time after the photoionizing lasers are switched on. As the size of the ion crystal grows, the fluorescence per ion increases due to the effect of rf micromotion for ions lying off axis, because they spend time closer to resonance with the red-detuned $397 \mathrm{~nm}$ cooling laser. However, for the range of crystal sizes in these experiments, the fluorescence per ion is roughly constant and we use the final fluorescence level as a measure of the total number of ions loaded. (The fluorescence was calibrated after a typical load by reducing the trap strength until the ions could be counted on the camera.) For each load, we wait a few seconds after the photoionizing lasers are switched off, to allow any hot ions to be cooled and to join the crystal, check that the ions are still crystalline using the camera, and then take the final number of ions $m$ divided by the duration $\tau$ of exposure to the photoionizing lasers as our measure of the loading rate $R$. We find that an error $\sqrt{m} / \tau$ is broadly consistent with variations in the loading rate taken under nominally identical conditions (see, for example, Fig. 6).

We have studied the loading rate as a function of the power $P_{423}$ and detuning $\Delta_{423}$ of the $423 \mathrm{~nm}$ laser, and of the power $P_{389}$ of the $389 \mathrm{~nm}$ laser. This was done at fixed oven current, to keep the atomic number density as constant as possible. For each measurement, the $397 \mathrm{~nm}$ fluorescence was allowed to increase to a level representing about 40 trapped ions, whereupon the photoionizing lasers were blocked. In practice, the number of ions loaded varied between 22(2) and 63(5); the length of photoionizing exposure was between $2 \mathrm{~s}$ and $600 \mathrm{~s}$. 


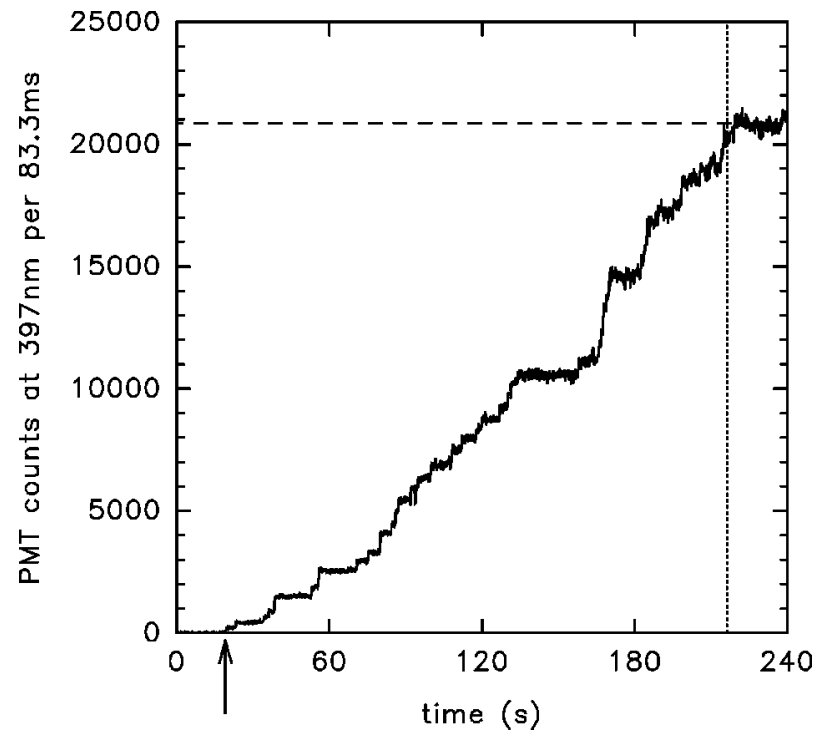

FIG. 5. Typical photoionization loading fluorescence curve. The $423 \mathrm{~nm}$ and $389 \mathrm{~nm}$ photoionizing lasers are switched on at $t=0$ and off at $t=216 \mathrm{~s}$ (dotted line). The $397 \mathrm{~nm}$ and $866 \mathrm{~nm}$ cooling lasers illuminate the ions continuously. Background counts due to scattered $397 \mathrm{~nm}$ light and (during the loading) $423 \mathrm{~nm}$ fluorescence and $389 \mathrm{~nm}$ light have been subtracted from the PMT signal. The step in $397 \mathrm{~nm}$ fluorescence when the first cold ion appears is arrowed. The large steps in the fluorescence are typical and may result from the details of the cooling dynamics as the number of ions in the trap increases. The dashed line indicates the final fluorescence level, which corresponds to 36(3) ions; the average loading rate of $0.17(3)$ ion/s is obtained by dividing this by the time for which the photoionizing lasers are switched on. The conditions were as follows: oven current $4.25 \mathrm{~A}, P_{423} \sim 6 \mu \mathrm{W}, \Delta_{423}$ $=0(5) \mathrm{MHz}, P_{389}=0.93(9) \mu \mathrm{W}, \Delta_{397}=-68(10) \mathrm{MHz}$.

Figure 6 shows the dependence of loading rate on $423 \mathrm{~nm}$ power and detuning. Note that the loading rate could in principle saturate at a different power level from the $423 \mathrm{~nm}$ fluorescence studied above, since photoionization is limited by the width of the $389 \mathrm{~nm}$ beam [the lifetime of the $4 s 4 p{ }^{1} P_{1}$ level, 4.49(4) ns, is much shorter than the time an atom spends in the laser beams, $\sim 0.2 \mu \mathrm{s}]$, whereas the fluorescence collected is limited by the imaging aperture. Nevertheless, we observe that the loading rate also starts to saturate above a power of $P_{423} \sim 10 \mu \mathrm{W}$ due to saturation of the upper state population. Given the power available in the 423 $\mathrm{nm}$ laser, the maximum loading rate could be made perhaps an order of magnitude larger by increasing the spot size at the trap to $1 \mathrm{~mm}$; spot sizes much larger than this, however, would start to reduce the isotope selectivity because of the increasing atomic beam divergence angle.

The loading rate as a function of $\Delta_{423}$ is of critical importance for isotope-selective loading. We expect the dependence to be modeled by a Voigt profile with similar Lorentzian width to that of the $423 \mathrm{~nm}$ fluorescence spectrum, but different Gaussian width because of the different atomic beam collimation angles for fluorescence detection and ion loading. The Lorentzian and Gaussian widths are not well determined if they are both floated, due to the noise in the data, so we fix the Lorentzian width at $39 \mathrm{MHz}$, from the
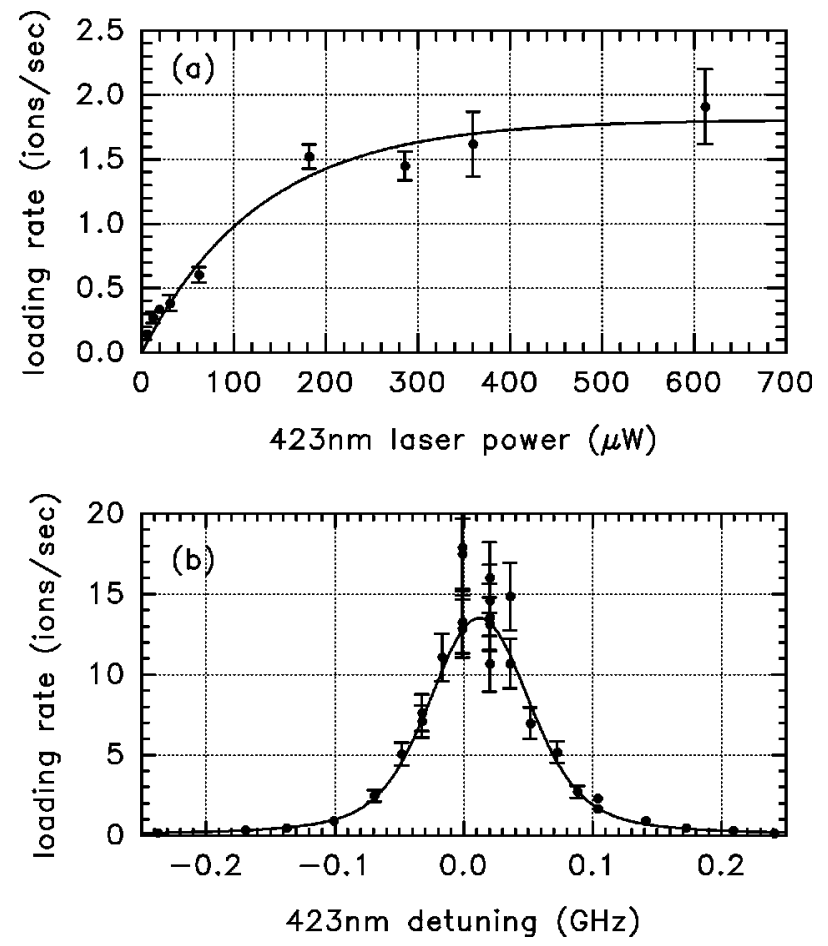

FIG. 6. (a) Photoionization loading rate as a function of $423 \mathrm{~nm}$ laser power, with oven current $4.25 \mathrm{~A}, \Delta_{423}=0(10) \mathrm{MHz}, P_{389}$ $=0.93(9) \mu \mathrm{W}, \Delta_{397}=-80(20) \mathrm{MHz}$. The loading rate starts to saturate for $P_{423} \gtrsim 10 \mu \mathrm{W}$. The curve is drawn to guide the eye. (b) Loading rate versus $423 \mathrm{~nm}$ detuning, with oven current $4.25 \mathrm{~A}$, $P_{423} \sim 6 \mu \mathrm{W}, P_{389}=80(8) \mu \mathrm{W}, \Delta_{397}=-97(10) \mathrm{MHz}$. A Voigt profile (solid line) is fitted to the data; the frequency offset, amplitude, and Gaussian width were floated while the Lorentzian width was fixed at $39 \mathrm{MHz}$ from the fit in Fig. 2. The FWHM of the fitted curve is $94 \mathrm{MHz}$. The frequency origin is taken from the fitted offset of a $423 \mathrm{~nm}$ fluorescence spectrum taken during the same run; the fitted profile here shows a blueshift of $12 \mathrm{MHz}$ which probably results from the slightly different collimation geometry relevant to loading.

fluorescence spectrum in Fig. 2, and find a fitted Gaussian width of $71 \mathrm{MHz}$. This Gaussian width is consistent with a beam collimation angle defined by the electrodes and an oven orifice of size $\sim 1 \mathrm{~mm}$. The consequent isotope selectivity expected is discussed in Sec. V A.

In contrast to the $423 \mathrm{~nm}$ bound-bound transition, no saturation effect is expected for the $389 \mathrm{~nm}$ bound-free ionizing transition, and this is verified for the $389 \mathrm{~nm}$ powers we can attain (Fig. 7): the dependence of the loading rate on $P_{389}$ is linear over two orders of magnitude. Significantly, there is no change in efficiency whether the $389 \mathrm{~nm}$ laser is running above or below lasing threshold, despite the fact that the spectral width is expected to be about five orders of magnitude larger $(\sim 3 \mathrm{~nm})$ below threshold. This implies a weak dependence on wavelength of the photoionization cross section in this region.

\section{Absolute photoionization efficiency}

We can deduce a lower limit for the photoionization cross section from the loading rate under conditions of near satu- 


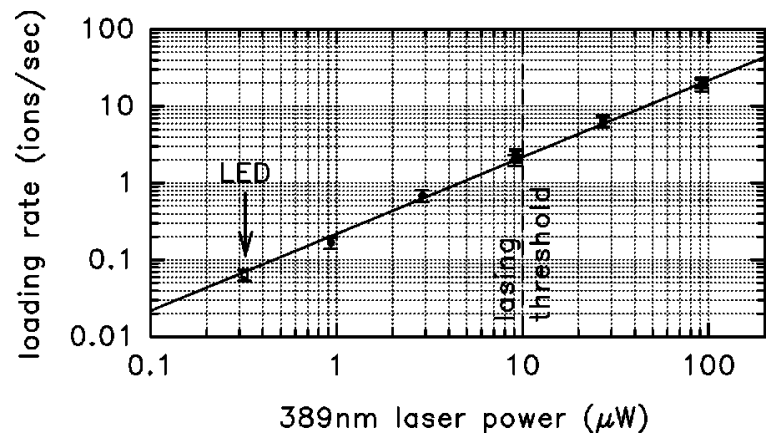

FIG. 7. Photoionization loading rate as a function of $389 \mathrm{~nm}$ laser power, with oven current $4.25 \mathrm{~A}, P_{423} \sim 6 \mu \mathrm{W}, \Delta_{423}$ $=0(5) \mathrm{MHz}, \Delta_{397}=-68(10) \mathrm{MHz}$. No significant difference in efficiency is observed even when the laser is well below lasing threshold (vertical dashed line), indicating that the photoionization cross section is independent of the spectral width of the laser. A straight line $(y \propto x)$ is fitted to the data. The single measurement with the ultraviolet LED in place of the $389 \mathrm{~nm}$ laser is also included (see Sec. IV D).

ration on the $423 \mathrm{~nm}$ transition, when we expect that nearly half the atoms are in the $4 s 4 p{ }^{1} P_{1}$ upper level (at least across that part of the $423 \mathrm{~nm}$ beam which overlaps the 389 $\mathrm{nm}$ beam). From the definition of the photoionization cross section $Q$, the number of atoms ionized per unit time is

$$
R=\frac{1}{2} a_{40} N Q l P_{389} / \varepsilon
$$

where $l=1.7(1) \mathrm{mm}$ is the length of the interaction region defined by the trap electrodes, $\left(\frac{1}{2} a_{40} N\right)$ the number density of ${ }^{40} \mathrm{Ca}$ atoms in the $4 s 4 p{ }^{1} P_{1}$ level, $P_{389}$ the $389 \mathrm{~nm}$ laser power, and $\varepsilon$ the $389 \mathrm{~nm}$ photon energy.

To minimize uncertainty in the number density due to variations in oven conditions, $N$ was determined shortly before measuring the loading rate and found to be 1.7(4) $\times 10^{11} \mathrm{~m}^{-3}$. At maximum $423 \mathrm{~nm}$ power, the estimated intensity at the center of the laser beam was 14(4) $I_{\text {sat }}$ which, for $\left|\Delta_{423}\right|<10 \mathrm{MHz}$, gives $n_{4 s 4 p}>0.48(1)$ within the halfintensity radius of the beam [Eq. (1)], justifying the assumption of saturation. The loading rate was measured to be $R$ $=4.9(9)$ ion $/ \mathrm{s}$ with $P_{389}=1.1(1) \mu \mathrm{W}$, giving a cross section $Q=170(60) \mathrm{Mb}$. This is a lower limit since it assumes that every ion produced is trapped and that the capture region of the trap extends across the full width of the atomic beam as far as the rf electrodes, but it lies in the anticipated range (Sec. IV A).

Alternatively, the loading rate can be expressed in terms of the probability $q$ per atom of being ionized (and trapped) as it crosses the interaction region:

$$
R=\frac{1}{2} a_{40} N q l w^{2} / t
$$

where $w^{2}$ is the cross-sectional area of the $389 \mathrm{~nm}$ beam (which limits the extent of the interaction region), and $t$ $\approx w / v$ is the time an atom of velocity $v$ spends in the interaction region. For the conditions above and the laser beam size $w \approx 0.1 \mathrm{~mm}$, we have $t \approx 0.2 \mu \mathrm{s}$ and find $q \approx 8 \times 10^{-7}$. The maximum $q$ we observed (with maximum powers in both the photoionizing lasers) was $q \approx 4 \times 10^{-5}$, and by eliminating the optical fiber and using the maximum available $389 \mathrm{~nm}$ power $\sim 2 \mathrm{~mW}$, we would in principle be able to obtain a maximum $q \approx 10^{-3}$, i.e., one in 1000 atoms crossing the interaction region would be loaded.

\section{Loading using an ultraviolet light-emitting diode}

The observation that the $389 \mathrm{~nm}$ laser is able to photoionize atoms just as efficiently below lasing threshold led us to experiment with an alternative light source for the second step of the photoionization, an ultraviolet light-emitting diode (LED). The device tested was a Nichia NSHU590 diode, with nominal output power $500 \mu \mathrm{W}$, peak wavelength 375 $\mathrm{nm}$, and spectral width $12 \mathrm{~nm}$. The chief advantages of this device are that it is about three orders of magnitude cheaper than a wavelength-selected ultraviolet laser diode, and does not require temperature and current stabilization. Measurements with a grating spectrograph indicated that the peak wavelength was in fact between $375 \mathrm{~nm}$ and $390 \mathrm{~nm}$, depending which part of the emission region was focused on the spectrograph slit, and the spectral width was $\sim 25 \mathrm{~nm}$; however, at least half of the spectral intensity lies in the range $\lambda<389.8 \mathrm{~nm}$ useful for photoionization. The main drawback of the LED is its extended emission region: it is difficult to focus a large fraction of the power into a region smaller than a few millimeters, unlike a laser beam.

When we first tested the LED in place of the $389 \mathrm{~nm}$ laser we were surprised to find that it was capable of loading ions into the trap while the $423 \mathrm{~nm}$ laser was blocked and the oven was switched off. A small number of ${ }^{40} \mathrm{Ca}^{+}$ions were loaded, together with several "dark" ions; at the same time we noticed that the stray dc electric field in the trap changed by $\sim 10 \%$, drifting gradually back to its former value over a period of an hour or so. Our interpretation is that the large $(\sim 5 \mathrm{~mm})$ patch of light from the LED was irradiating the rf electrodes, ablating and photoionizing calcium and other ions from the surface where they had previously been deposited by the atomic beam. Photoelectric emission could be responsible for charging insulating patches on the electrodes, causing the stray field to change temporarily before the charge leaks away (the photoelectric cutoff wavelength for calcium is $433 \mathrm{~nm}$ ). Surface ablation is a well-known method of loading ion traps [29], although it is perhaps surprising at the low light intensities involved here.

To avoid this problem, we imaged the LED onto a 200 $\mu \mathrm{m}$ pinhole, which was reimaged at approximately $1: 1$ magnification into the trap region, and overlapped with the 423 $\mathrm{nm}$ beam. This eliminated loading of non- ${ }^{40} \mathrm{Ca}^{+}$ions and effects on the stray field, but only $0.32(3) \mu \mathrm{W}$ of ultraviolet power was then available at the trap. The loading rate under these conditions, 0.06(1) ion/s, is plotted in Fig. 7 and is similar to that expected with comparable $389 \mathrm{~nm}$ laser power. This rate is not sufficient for loading the rarer isotopes, where it is necessary to beat losses due to charge exchange (Sec. V B), but is perfectly adequate for loading small crystals of ${ }^{40} \mathrm{Ca}^{+}$. We note that high-power $(\sim 150$ $\mathrm{mW}$ ) ultraviolet LEDs are also available, although their cost approaches that of a laser diode. 


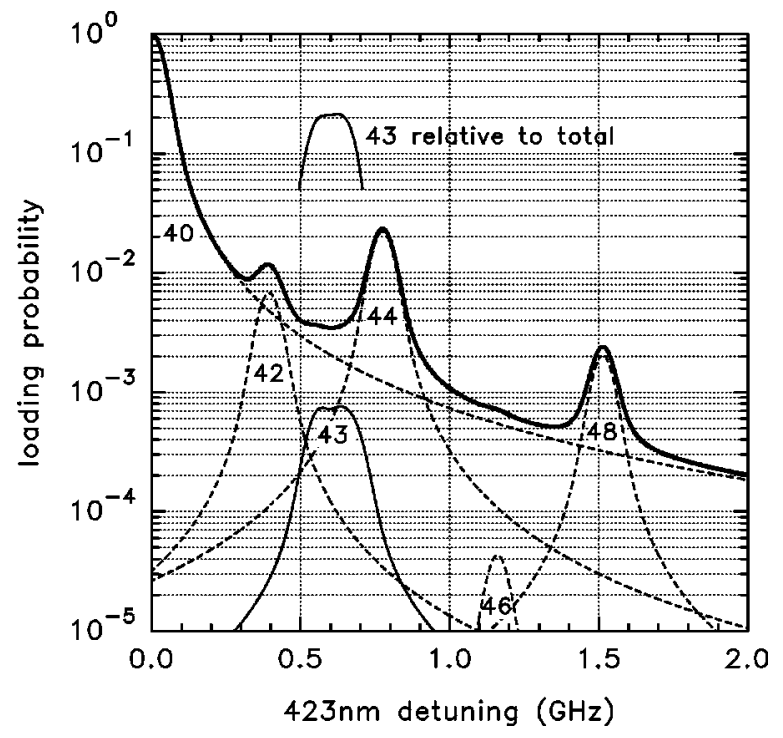

FIG. 8. Probability of loading different calcium isotopes as a function of detuning of the $423 \mathrm{~nm}$ laser, normalized to 1 for ${ }^{40} \mathrm{Ca}^{+}$ at zero detuning. The curves are generated using Voigt profiles with the same width parameters as the fit in Fig. 6(b) (except that the Gaussian width is adjusted to take account of the different isotopic masses). The dashed curves for the even isotopes, and the solid curve for ${ }^{43} \mathrm{Ca}^{+}$(the three hyperfine components have been summed), are labeled by the mass numbers. The thick solid curve is the sum of the contributions from all the isotopes. The detached solid curve gives the probability of loading ${ }^{43} \mathrm{Ca}^{+}$relative to the total loading probability at the same detuning, plotted in the region where this quantity is greater than 0.05 .

\section{ISOTOPE-SELECTIVE LOADING}

\section{A. Expected selectivity}

From the data in Fig. 6(b) we expect good isotope selectivity, because the net FWHM, $94 \mathrm{MHz}$, is small compared with the isotope shifts in the $423 \mathrm{~nm}$ transition. This discrimination has to be set against the very low abundance of some isotopes. In Fig. 8 we plot the calculated probability of loading the different isotopes as a function of detuning $\Delta_{423}$, based on the fitted Voigt profile in Fig. 6(b). We regard the loading as "isotope selective" for those isotopes where there exists a detuning at which one is more likely to load the desired isotope than any other; it can be seen from Fig. 8 that this is the case for all isotopes except ${ }^{43} \mathrm{Ca}^{+}$and ${ }^{46} \mathrm{Ca}^{+}$. The most demanding case is ${ }^{46} \mathrm{Ca}^{+}$, where the maximum achievable loading probability, relative to the total loading probability, is only $6.0 \%$. The relative probability of loading ${ }^{43} \mathrm{Ca}^{+}$is also plotted in the figure: it reaches a maximum of $21 \%$ at a detuning of $0.623 \mathrm{GHz}$. This compares with a theoretical maximum of $33 \%$ which would be possible with a Lorentzian width equal to the natural linewidth and negligible Gaussian width; thus not a great deal would be gained by efforts to further collimate the atomic beam.

The detunings of the Doppler-cooling lasers also affect the selectivity, due to the isotope shifts in the ionic transitions (Table I). For example, to cool ${ }^{42} \mathrm{Ca}^{+}$efficiently, the $397 \mathrm{~nm}$ laser needs to be detuned $\sim 350 \mathrm{MHz}$ to the blue of the ${ }^{40} \mathrm{Ca}^{+}$transition, which will tend to heat ${ }^{40} \mathrm{Ca}^{+}$ions and expel them from the trap, thus enhancing the selectivity.

\section{B. Charge-exchange loss}

It is possible for an ion ${ }^{X} \mathrm{Ca}^{+}$which has been loaded into the trap to undergo charge exchange with a neutral atom in the atomic beam; since the overwhelming probability is that the neutral atom is ${ }^{40} \mathrm{Ca}$ the process is usually

$$
{ }^{X} \mathrm{Ca}^{+}+{ }^{40} \mathrm{Ca} \rightarrow{ }^{X} \mathrm{Ca}+{ }^{40} \mathrm{Ca}^{+},
$$

and the original ion in the trap is replaced by ${ }^{40} \mathrm{Ca}^{+}$. The charge-exchange rate is proportional to the number density in the atomic beam. This process is a limitation for loading more than one ion of a rare isotope: if the loss rate through charge exchange exceeds the loading rate, then rare ions already trapped are replaced by ${ }^{40} \mathrm{Ca}^{+}$faster than subsequent ones can be loaded. If the charge-exchange rate is sufficiently fast, then rare ions will not even be cooled before they are replaced, and the trap will fill up with ${ }^{40} \mathrm{Ca}^{+}$before any fluorescence signal from the rare ions is seen.

In practice we find that, providing one is not attempting to load large crystals, charge exchange is only a limitation for the rarest isotope ${ }^{46} \mathrm{Ca}^{+}$. For the typical loading parameters used for loading ${ }^{43} \mathrm{Ca}^{+}$, for example, the loading rate is $\sim 1$ ion/s while the lifetime against loss due to charge exchange is $\sim 1$ min. ${ }^{46} \mathrm{Ca}^{+}$is about 30 times less abundant, so we expect the loss rate to approach the loading rate under the same conditions.

We note that the charge-exchange process was used by Mortensen et al. [2] to demonstrate isotope-selective loading, but that they were not attempting to retain and to lasercool the different isotopes; instead they relied on charge exchange to replace every isotope by ${ }^{40} \mathrm{Ca}^{+}$and used the resulting ${ }^{40} \mathrm{Ca}^{+}$fluorescence as a measure of the original load size.

\section{Even isotopes}

Loading one of the even isotopes consists of choosing the $423 \mathrm{~nm}$ detuning for maximum selectivity and setting the appropriate detunings of the $397 \mathrm{~nm}$ and $866 \mathrm{~nm}$ lasers used for Doppler cooling. After loading, we can check for the presence of other isotopes by looking for fluorescence using different detunings of the cooling lasers (the lasers are blocked while the detunings are changed, to avoid possible heating). Phase-sensitive detection with a chopped $866 \mathrm{~nm}$ beam can be used to detect very small fluorescence signals above scattered $397 \mathrm{~nm}$ background light.

As expected from Fig. 8 we can load small, pure crystals of ${ }^{44} \mathrm{Ca}^{+}$and ${ }^{48} \mathrm{Ca}^{+}$(and of course ${ }^{40} \mathrm{Ca}^{+}$) without difficulty. Predicted peak loading probabilities of ${ }^{44} \mathrm{Ca}^{+}$and ${ }^{48} \mathrm{Ca}^{+}$are $94 \%$ and $85 \%$, respectively. Practical loading rates can be achieved in spite of the small abundances by using maximum $389 \mathrm{~nm}$ power $(\sim 100 \mu \mathrm{W})$ and by increasing the oven current. We concentrate here on the more challenging cases of ${ }^{42} \mathrm{Ca}^{+}$(which lies closest to ${ }^{40} \mathrm{Ca}^{+}$) and ${ }^{46} \mathrm{Ca}^{+}$(which is extremely rare).

The maximum expected probability of loading ${ }^{42} \mathrm{Ca}^{+}$is $58 \%$, at a detuning $\Delta_{423}=0.394 \mathrm{GHz}$, with ${ }^{40} \mathrm{Ca}^{+}$by far the 
dominant impurity. However, when we loaded using this detuning, we obtained a crystal consisting of $\sim 19{ }^{42} \mathrm{Ca}^{+}$ions and only $\sim 5{ }^{40} \mathrm{Ca}^{+}$ions, i.e., a loading efficiency of about $80 \%$. We attribute this to the mechanism mentioned above, that the $397 \mathrm{~nm}$ laser was blue-detuned from the ${ }^{40} \mathrm{Ca}^{+}$transition by about $350 \mathrm{MHz}$, thus tending to heat this species and expel it from the trap; the ${ }^{40} \mathrm{Ca}^{+}$ions are only cooled sympathetically by the ${ }^{42} \mathrm{Ca}^{+}[30]$. This mixed-species crystal also illustrates the typical charge-exchange rates: after $\sim 20$ min, with the photoionizing lasers blocked but the oven left on at $4.25 \mathrm{~A}$, the number of ${ }^{40} \mathrm{Ca}^{+}$ions had increased to $\sim 18$ and there was only a very low signal visible at the ${ }^{42} \mathrm{Ca}^{+}$detunings; after a further $30 \mathrm{~min}$, there was no detectable signal from ${ }^{42} \mathrm{Ca}^{+}$. This indicates that the lifetime (per ion) against loss by charge exchange is $\sim 17 \mathrm{~min}$, for a number density in the atomic beam of $9(2) \times 10^{10} \mathrm{~m}^{-3}$. The limited isotope selectivity achievable could be overcome by selective heating methods $[13,14]$ to obtain a pure crystal of ${ }^{42} \mathrm{Ca}^{+}$.

The $0.004 \%$ abundant ${ }^{46} \mathrm{Ca}^{+}$is the most difficult isotope to load, with an expected peak loading probability of $6.0 \%$ (neglecting enhancement by heating as observed for ${ }^{42} \mathrm{Ca}^{+}$). To achieve the maximum photoionization rate without excessive saturation broadening of the $423 \mathrm{~nm}$ transition, we set $P_{423} \sim 180 \mu \mathrm{W}$; we used maximum $389 \mathrm{~nm}$ power $(\sim 100$ $\mu \mathrm{W})$ and increased the oven current to 5.0 A. Using the measured loading rates for ${ }^{40} \mathrm{Ca}^{+}$and the number density data we expect a ${ }^{46} \mathrm{Ca}^{+}$loading rate of $\sim 0.1 \mathrm{ion} / \mathrm{s}$, with a sacrifice of a factor $\sim 3$ in selectivity because of the high 423 $\mathrm{nm}$ power. Under these conditions, a fluorescence signal was detected after $\sim 17 \mathrm{~s}$ of exposure to the photoionizing lasers. The oven was switched off immediately to prevent charge exchange. The ions could not be made to crystallize, however, indicating that too many impurity ions were present to be cooled sympathetically by the ${ }^{46} \mathrm{Ca}^{+}$. We therefore applied a "tickle" voltage to one of the trap end caps, close to the axial resonance frequency, which is less likely to expel the directly cooled ${ }^{46} \mathrm{Ca}^{+}$than other isotopes. Crystallization was then observed, Fig. 9, and camera images implied that the crystal consisted of a single ${ }^{46} \mathrm{Ca}^{+}$ion and several impurity ions (most probably ${ }^{40} \mathrm{Ca}^{+}$). The observed isotope shift in the $866 \mathrm{~nm}$ transition was $-6.4(2) \mathrm{GHz}$; although this particular shift has not been measured before, it is in agreement with that expected (Table I) and thus confirms the identity of the ion.

\section{Odd isotope}

The laser requirements for Doppler-cooling ${ }^{43} \mathrm{Ca}^{+}$are more demanding than for the even isotopes because of the hyperfine structure, Fig. 1(b). In particular, a separate repumper is necessary on one of the ultraviolet transitions because of the large $(3.2 \mathrm{GHz})$ hyperfine splitting between the $S_{1 / 2}^{3}$ and $S_{1 / 2}^{4}$ levels (where the superscript denotes $F$ ).

We choose the $393 \mathrm{~nm} S_{1 / 2}^{4} \leftrightarrow P_{3 / 2}^{5}$ transition for cooling, both because it is an intrinsically strong $F \leftrightarrow F+1$ transition and because (thanks to the inverted hyperfine structure) it is blue-detuned from the $S_{1 / 2} \leftrightarrow P_{3 / 2}$ transitions in all the even isotopes (inset of Fig. 1). This second fact means that any

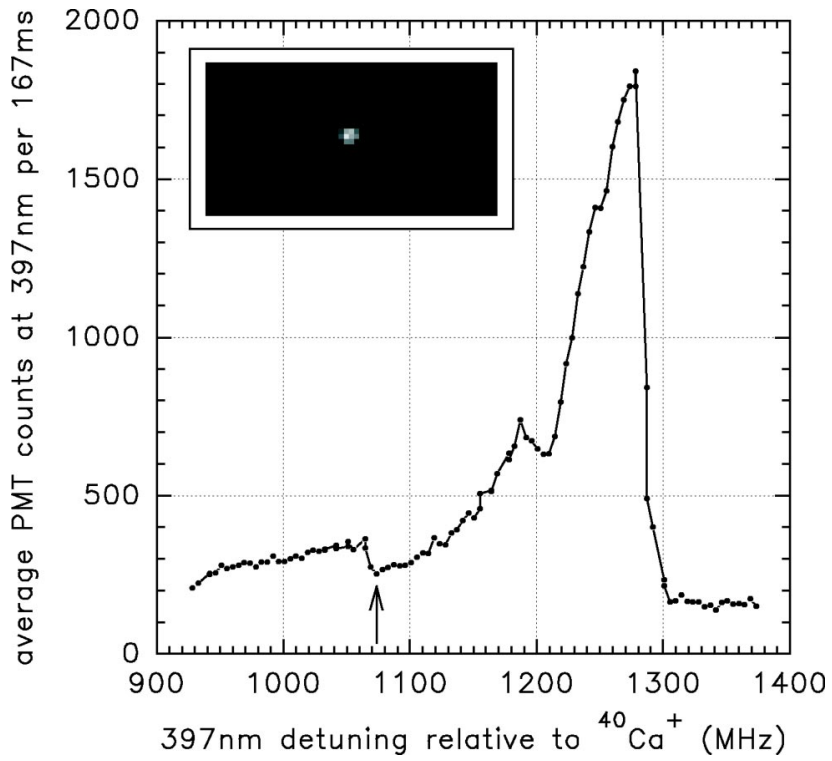

FIG. 9. Fluorescence signal from a single ${ }^{46} \mathrm{Ca}^{+}$ion, trapped in the company of several "dark" ions (probably ${ }^{40} \mathrm{Ca}^{+}$), as a function of $397 \mathrm{~nm}$ detuning relative to ${ }^{40} \mathrm{Ca}^{+}$. Three scans were averaged to produce the plot. The high-frequency "edge" in the fluorescence curve, characteristic of scanning past the resonant frequency, has been assigned the known isotope shift of $1287 \mathrm{MHz}$. The first dip in the fluorescence (arrowed) is the point at which the ions crystallize, and the single ${ }^{46} \mathrm{Ca}^{+}$ion becomes visible on the camera (inset). The second dip, at $\sim 1200 \mathrm{MHz}$, is probably due to a two-photon dark resonance between the $866 \mathrm{~nm}$ and $397 \mathrm{~nm}$ transitions [31]. The crystallization dip, and the movement of the single ${ }^{46} \mathrm{Ca}^{+}$ion between discrete positions in the trap after heating and recrystallization, indicate the presence of other ions.

other isotopes that are loaded will tend to be heated. Furthermore, this transition is nearly cycling (apart from the $\sim 6 \%$ decay route to the $D$ levels and off-resonant driving of the $160 \mathrm{MHz}$-detuned $S_{1 / 2}^{4} \leftrightarrow P_{3 / 2}^{4}$ component), so that the second ultraviolet laser needed for repumping out of the $S_{1 / 2}^{3}$ level can still repump efficiently at low intensities: this is an advantage since it is necessarily red detuned relative to the even isotopes and can thus cool them. We choose to use the $397 \mathrm{~nm} S_{1 / 2}^{3} \leftrightarrow P_{1 / 2}^{4}$ transition for this repumping.

Repumping from the $D_{3 / 2}$ and $D_{5 / 2}$ manifolds is accomplished with the $850 \mathrm{~nm}$ and $854 \mathrm{~nm}$ lasers, respectively: the high intensities available in these beams mean that they can repump adequately in spite of the hyperfine structure, which spans $\sim 400 \mathrm{MHz}$ in each transition.

To load we set the appropriate detunings of these four lasers relative to ${ }^{40} \mathrm{Ca}^{+}$and set $\Delta_{423} \approx 600 \mathrm{MHz}$. The $423 \mathrm{~nm}$ power is typically $\sim 10 \mu \mathrm{W}$, to avoid saturation broadening, and the $389 \mathrm{~nm}$ power maximized $(\sim 100 \mu \mathrm{W})$ for fastest loading. Under these conditions and with an oven current of $5.0 \mathrm{~A}$, the loading rate is $\sim 1 \mathrm{ion} / \mathrm{s}$ and the lifetime against loss through charge exchange $\sim 1 \mathrm{~min}$. This gives time to detect a single ion of ${ }^{43} \mathrm{Ca}^{+}$and switch the oven off before it is replaced by one of ${ }^{40} \mathrm{Ca}^{+}$. With this procedure, we can reliably load single ions of ${ }^{43} \mathrm{Ca}^{+}$and only rarely find that we have loaded ${ }^{40} \mathrm{Ca}^{+}$as well; this indicates that we achieve significant enhancement over the estimated maximum load- 


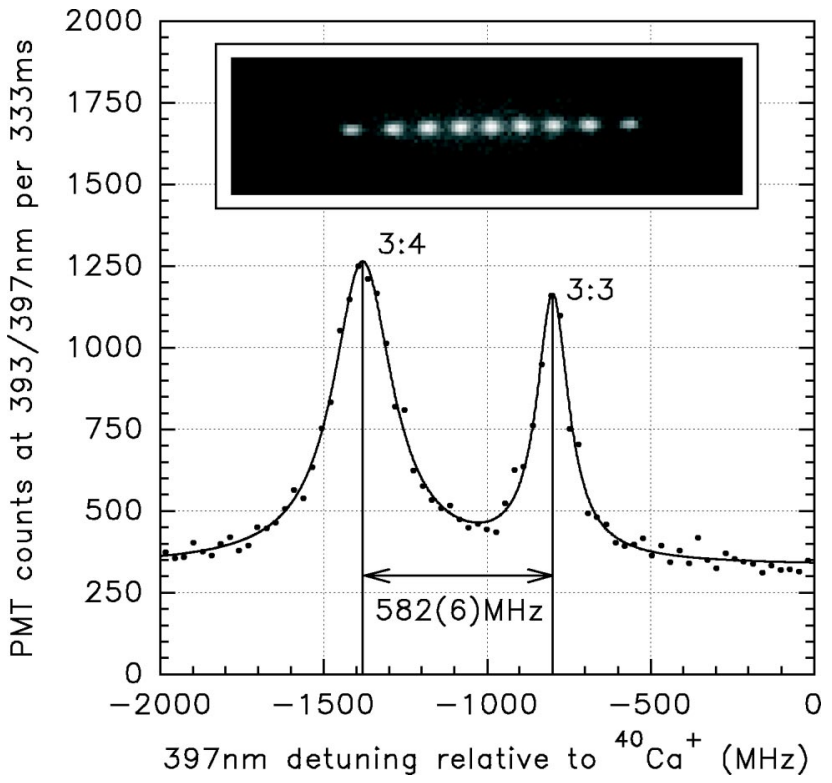

FIG. 10. Fluorescence signal from a single ${ }^{43} \mathrm{Ca}^{+}$ion as the frequency of the $397 \mathrm{~nm}$ ultraviolet repumping laser is scanned. The $397 \mathrm{~nm}$ intensity was reduced to $\sim 0.2 \mathrm{~mW} / \mathrm{mm}^{2}$ for this scan, to reduce the widths of the peaks. A double Lorentzian is fitted to the data: the separation between the two components is $582(6) \mathrm{MHz}$ (where a $1 \%$ error is allowed in deducing the frequency scale from a confocal cavity of $300 \mathrm{MHz}$ free spectral range), in excellent agreement with the known hyperfine splitting in the $P_{1 / 2}$ level of $581 \mathrm{MHz}$. The $S_{1 / 2}^{3} \leftrightarrow P_{1 / 2}^{4}$ component has been assigned the known shift of $-1381 \mathrm{MHz}$ relative to ${ }^{40} \mathrm{Ca}^{+}$. Inset: A pure crystal of nine ${ }^{43} \mathrm{Ca}^{+}$ions, loaded using periodic blocking of the $397 \mathrm{~nm}$ repumper to remove even-isotope impurity ions, as described in the text. The separation between the closest ions is 13(1) $\mu \mathrm{m}$.

ing probability of only $21 \%$, presumably because ${ }^{40} \mathrm{Ca}^{+}$ions are heated by the $393 \mathrm{~nm}$ laser and expelled from the trap. The maximum fluorescence rate detected from a single ${ }^{43} \mathrm{Ca}^{+}$ion was $23000 \mathrm{~s}^{-1}$, compared with typically 32000 $\mathrm{s}^{-1}$ for ${ }^{40} \mathrm{Ca}^{+}$(a reduction attributable to less efficient repumping from the $D$ states).

To confirm the identity of the first single ion we loaded by this method, we scanned the frequency of the $397 \mathrm{~nm}$ repumper: at low intensity, the two hyperfine components $S_{1 / 2}^{3} \leftrightarrow P_{1 / 2}^{4}$ and $S_{1 / 2}^{3} \leftrightarrow P_{1 / 2}^{3}$ are well resolved, Fig. 10, and have the expected separation. As a further check, the axial resonance frequency was measured and compared with that of ${ }^{40} \mathrm{Ca}^{+}$, giving a mass number of $42.8(2)$.

If the photoionizing lasers and oven are left on for longer we find that we load crystals containing about $50 \%{ }^{43} \mathrm{Ca}^{+}$ and $50 \%{ }^{40} \mathrm{Ca}^{+}$. This may occur either because of charge exchange, or because once ${ }^{43} \mathrm{Ca}^{+}$is trapped and cooled, it can cool ${ }^{40} \mathrm{Ca}^{+}$sympathetically in spite of the heating effect of the $393 \mathrm{~nm}$ laser. We discovered that it was possible to purify such mixed crystals by temporarily blocking the 397 $\mathrm{nm}$ ultraviolet repumper; when this laser is blocked a small fluorescence signal is still visible from ${ }^{43} \mathrm{Ca}^{+}$(because of off-resonant repumping on $S_{1 / 2}^{3} \leftrightarrow P_{3 / 2}^{2 ; 3 ; 4}$ by the $3 \mathrm{GHz}-$ detuned $393 \mathrm{~nm}$ laser) but there is no longer any laser capable of cooling ${ }^{40} \mathrm{Ca}^{+}$directly. We assist the ${ }^{40} \mathrm{Ca}^{+}$heating by switching on the $866 \mathrm{~nm}$ repumper, tuned to be resonant with the ${ }^{40} \mathrm{Ca}^{+}$transition. One might expect the Coulomb coupling between the different species to make this purification process rather inefficient, but we found, on the contrary, that it always seems successful at removing the ${ }^{40} \mathrm{Ca}^{+}$ions without loss of any ${ }^{43} \mathrm{Ca}^{+}$. For example, a mixed crystal consisting of three ${ }^{43} \mathrm{Ca}^{+}$ions and eight ${ }^{40} \mathrm{Ca}^{+}$ions was purified completely by blocking the $397 \mathrm{~nm}$ repumper for $\sim 8$ s. The technique can also be applied during loading: with the oven and photoionizing lasers on, we watch the ${ }^{43} \mathrm{Ca}^{+}$ ions being loaded on the camera and, as soon as there is evidence of "dark ions" in the trap, block the $397 \mathrm{~nm}$ repumper for a few seconds to purify the crystal, then unblock it to continue loading. In this way, pure ${ }^{43} \mathrm{Ca}^{+}$crystals of arbitrary size can be loaded; the inset of Fig. 10 shows a nine-ion linear crystal.

\section{PHOTOIONIZATION AND ELECTRON BOMBARDMENT COMPARED}

The limitations of electron bombardment ionization are discussed in [3]. A significant problem in our apparatus was the effect of firing the electron gun on the stray electric fields in the trap. After loading, the static field necessary to compensate the micromotion of the ions was found to decay exponentially with a time constant $\sim 6 \mathrm{~h}$. For the typical load shown in Fig. 11(a) the vertical stray field would not have been stable to the $1 \mathrm{~V} / \mathrm{m}$ resolution of our compensation method until $\sim 28 \mathrm{~h}$ after the trap was loaded. We ascribe this drift to charge on insulating parts of the trap structure which decays with time, and have not observed this systematic decay after photoionization loading. On longer time scales, between loads, we observed that the vertical stray field changed by up to $\sim 200 \mathrm{~V} / \mathrm{m}$; this is compared with recent photoionization compensation data in Fig. 11(b). The cause of these long-term changes in the stray field could have been changes in the distribution of material deposited on the trap electrodes when the oven or electron gun was fired: calcium deposited on the stainless steel rf electrodes could give rise to static fields of this magnitude, due to the difference in work functions between these two metals.

This highlights a further issue: based on the work of the NIST Ion Storage group, we expect that material deposited onto the trap electrodes from the oven and/or electron gun will contribute significantly to motional heating of trapped ions in the sub-Doppler cooling regime relevant to quantum logic experiments $[4,32]$. The estimates of calcium number density and temperature in the atomic beam given in Sec. III C above imply a flux of $\sim 4 \times 10^{9}$ (atom/s) $/ \mathrm{mm}^{2}$ on a surface normal to the atomic beam, at the $6 \mathrm{~A}$ oven current which was necessary for loading the trap using electron bombardment. For the same loading rate, this can be reduced by over four orders of magnitude using photoionization. Furthermore, the same photoionization loading rate can be maintained even when using a well-collimated atomic beam designed to prevent deposition of atoms on the trap structure.

With maximum power in both photoionizing lasers, we measured a loading rate of 1.6(2) ion/s at an oven current of 3.25 A. This compares with an electron bombardment load- 

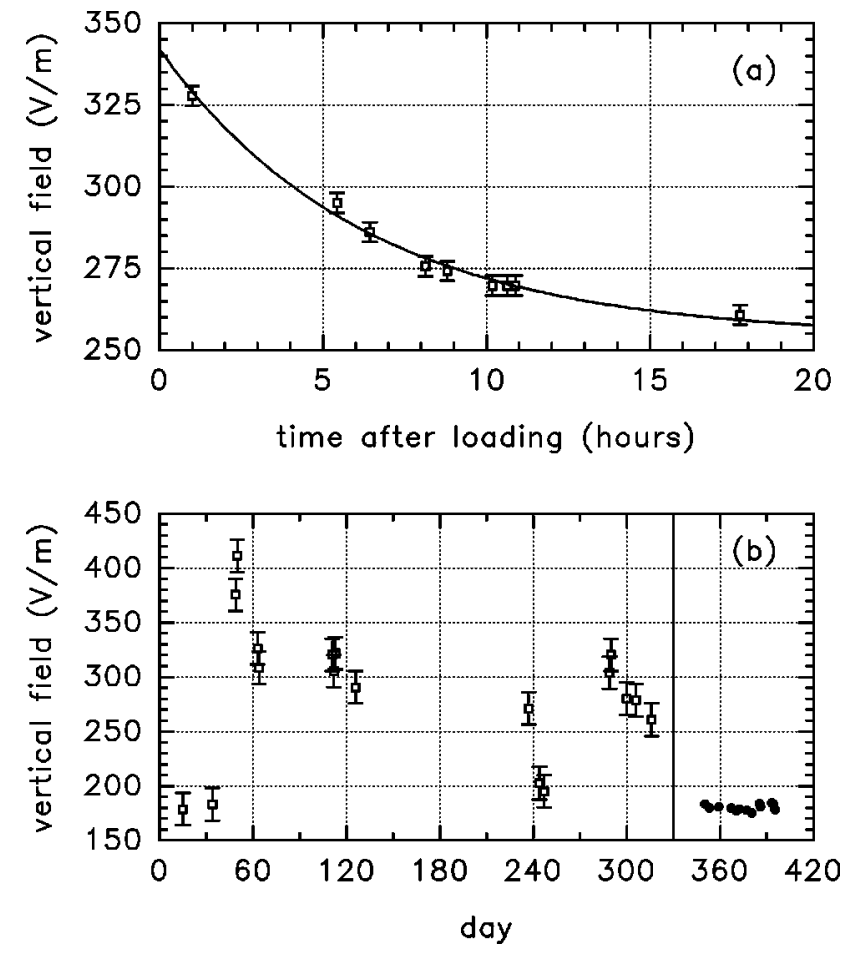

FIG. 11. (a) Drift of the stray field versus time after loading with electron bombardment ionization. The magnitude of the vertical field required to compensate horizontal ion micromotion is plotted. The micromotion compensation here was performed by minimizing the width of the $S_{1 / 2} \leftrightarrow P_{1 / 2}$ spectrum; we assign an error of \pm 3 $\mathrm{V} / \mathrm{m}$. An exponential decay function is fitted to the data (solid line); this has time constant 6.3(7) h and amplitude 88(4) V/m. No such drift is visible after loading by photoionization, at the $1 \mathrm{~V} / \mathrm{m}$ level. (b) Variation of the steady-state stray field versus time, over the course of nearly a year for electron bombardment loading (squares, left) and over the course of 45 days for photoionization loading (circles, right). Each point is taken from a different load. For the electron bombardment data the error bar represents the estimated additional drift possible after the final compensation measurement. For the photoionization data, the estimated error in compensating the stray field is $1 \mathrm{~V} / \mathrm{m}$; the standard deviation of the data set is 2.8 $\mathrm{V} / \mathrm{m}$, indicating some residual systematic variation (which may be due to the remaining atomic beam flux, or mechanical variations in the trap geometry over time).

ing rate of $\sim 1$ ion/s at an oven current of 6.0 A. Extrapolating the number density data of Fig. 4(a) to 6.0 A oven current indicates that photoionization is a factor of $\sim 10^{4}$ times more efficient than our electron bombardment ionization setup. A further two orders of magnitude enhancement of the loading efficiency in this apparatus would be available by (i) increasing the spot sizes of the photoionizing lasers to reduce saturation effects on the $423 \mathrm{~nm}$ transition and (ii) eliminating the optical fiber in the $389 \mathrm{~nm}$ beam. We note, however, that the per atom efficiency of photoionization trap loading can be approached by techniques of surface ionization [33].

The photoionization method overcomes the limitations of electron bombardment ionization: the species and isotope selectivity ensure that unwanted ions are not trapped, and furthermore allow studies of rare isotopes without enriched sources; there is negligible effect due to the loading on the stray fields (at the level of $\sim 1 \mathrm{~V} / \mathrm{m}$ in this trap); it works well irrespective of radial trap strength [3], so that compensation of stray fields for different trap conditions is unnecessary; the high efficiency of photoionization means that the flux of the atomic beam can be attenuated by many orders of magnitude, which immensely reduces problems associated with deposition of material on the trap structure. These advantages must be weighed against the complexities of two additional lasers, compared with an electron gun inside the vacuum system.

\section{CONCLUSION}

We have presented quantitative studies of photoionization ion-trap loading for calcium ions, using the fluorescence from the neutral atoms to estimate the absolute efficiency of this process. We find that, in our trap, this loading method is around four orders of magnitude more efficient than conventional electron impact ionization, comparably to the results of Gulde et al. [3], and does not give rise to stray electric fields. We have shown that the setup of Gulde et al. may be further simplified by eliminating frequency stabilization for the $389 \mathrm{~nm}$ laser and, if high loading rates are not required, by replacing this laser with a light-emitting diode. We have optimized the photoionization procedure for the purposes of isotope selection, demonstrating trapping of all the naturally occurring isotopes from a natural abundance source.

The odd isotope ${ }^{43} \mathrm{Ca}^{+}$is of particular interest for experiments in quantum information processing and optical frequency standards, and we have demonstrated the ability to load pure crystals of this ion. We note, however, that an enriched source would still be advantageous for work with this isotope, since it would reduce any remaining deposition of material from the atomic beam on the trap electrodes to the minimum possible. An interesting feature of photoionization compared with electron bombardment ionization is that the laser beams allow it to be spatially selective: this could be useful for loading specific traps in proposed quantum computing architectures involving microtrap arrays [34]. The spatial selectivity could be extended to three dimensions by crossing the $423 \mathrm{~nm}$ and $389 \mathrm{~nm}$ beams. The isotope selectivity would also be invaluable for loading "refrigerator" ions [35] in schemes where continuous cooling is provided by one isotope, while quantum logic operations are carried out using another isotope: following the estimate in [29], the decoherence rate due to off-resonant photon scattering, of a ${ }^{43} \mathrm{Ca}^{+}$qubit being cooled sympathetically by a ${ }^{42} \mathrm{Ca}^{+}$refrigerator ion is $\sim 0.1 / \mathrm{s}$ for a trap with a heating rate of $10^{3}$ motional quanta per second [4].

Finally, in comparison with the work of Mortensen et al., it is clear that the $272 \mathrm{~nm} 4 s^{2}{ }^{1} S_{0} \leftrightarrow 4 s 5 p{ }^{1} P_{1}$ transition is much more favorable for isotope-selective ionization of calcium than the $423 \mathrm{~nm}$ transition used in this work, since it possesses a narrower natural width, 8.5(5) $\mathrm{MHz}$ [17], and isotope shifts about a factor of 2 larger [2]. However, this scheme requires a significantly more complex laser source than the blue diode laser used here and is somewhat less efficient since it involves excitation deeper into the continuum. An unfortunate circumstance at the time of writing is 
the unavailability of further diodes at $423 \mathrm{~nm}$ from Nichia Corporation but we are optimistic that, with blue laser diodes under development by several other manufacturers and research laboratories, the situation will improve.

\section{ACKNOWLEDGMENTS}

We are extremely grateful to Dr. Hugo van der Hart and Claire McKenna for performing calculations of the photoionization cross section. We would also like to thank the iontrapping group of Professor Dr. Rainer Blatt at Universität
Innsbruck for many useful discussions, Dr. Charles Donald for compiling the electron bombardment ionization data in Fig. 11, and Wolfgang Kemp at Toptica Photonics for helpful advice. Graham Quelch contributed invaluable technical assistance. The research is supported by the EPSRC, ARDA (Grant No. P-43513-PH-QCO-02107-1), and the E.U. QGATES network. M.M. acknowledges support from the Association of Commonwealth Universities. D.M.L. acknowledges support from the Royal Society and wishes to thank Dr. Bruce Warrington for SEVENSEGMENTDISPLAY software development.
[1] N. Kjaergaard, L. Hornekaer, A. M. Thommesen, Z. Videsen, and M. Drewsen, Appl. Phys. B: Lasers Opt. 71, 207 (2000).

[2] A. Mortensen, J. Lindballe, I. S. Jensen, D. Voigt, and M. Drewsen, in XVIII International Conference on Atomic Physics (Poster Presentation Abstracts) (World Scientific, Singapore, 2002), p. 253.

[3] S. Gulde, D. Rotter, P. Barton, F. Schmidt-Kaler, R. Blatt, and W. Hogervorst, Appl. Phys. B: Lasers Opt. 73, 861 (2001).

[4] Q. A. Turchette et al., Phys. Rev. A 61, 063418 (2000).

[5] A. B. Mundt, A. Kreuter, C. Russo, C. Becher, D. Leibfried, J. Eschner, F. Schmidt-Kaler, and R. Blatt, Appl. Phys. B: Lasers Opt. 76, 117 (2003).

[6] M. Keller, B. Lange, K. Hayasaka, W. Lange, and H. Walther, Appl. Phys. B: Lasers Opt. 76, 125 (2003).

[7] A. M. Steane, Appl. Phys. B: Lasers Opt. 64, 623 (1997).

[8] D. M. Lucas, C. J. S. Donald, J. P. Home, M. J. McDonnell, A. Ramos, D. N. Stacey, J.-P. Stacey, A. M. Steane, and S. C. Webster, Philos. Trans. R. Soc. London, Ser. A 361, 1401 (2003).

[9] F. Schmidt-Kaler, S. Gulde, M. Riebe, T. Deuschle, A. Kreuter, G. Lancaster, C. Becher, J. Eschner, H. Häffner, and R. Blatt, J. Phys. B 36, 623 (2003).

[10] F. Plumelle and M. Desaintfusien, IEEE Trans. Instrum. Meas. 42, 462 (1993).

[11] M. G. Boshier, G. P. Barwood, G. Huang, and H. A. Klein, Appl. Phys. B: Lasers Opt. 71, 51 (2000).

[12] R. L. Cline (private communication).

[13] R. Alheit, K. Enders, and G. Werth, Appl. Phys. B: Lasers Opt. 62, 511 (1996).

[14] K. Toyoda, H. Kataoka, Y. Kai, A. Miura, M. Watanabe, and S. Urabe, Appl. Phys. B: Lasers Opt. 72, 327 (2001).

[15] W. Nörtershäuser, K. Blaum, K. Icker, P. Müller, A. Schmitt, K. Wendt, and B. Wiche, Eur. Phys. J. D 2, 33 (1998).

[16] W. Nörtershäuser, N. Trautmann, K. Wendt, and B. A. Bushaw,
Spectrochim. Acta, Part B 53, 709 (1998).

[17] J. Mitroy, J. Phys. B 26, 3703 (1993).

[18] P. Barton, C. J. S. Donald, D. M. Lucas, D. A. Stevens, A. M. Steane, and D. N. Stacey, Phys. Rev. A 62, 032503 (2000).

[19] T. B. Coplen et al., Pure Appl. Chem. 74, 1987 (2002).

[20] A. M. Mårtensson-Pendrill, A. Ynnerman, H. Warston, L. Vermeeren, R. E. Silverans, A. Klein, R. Neugart, C. Schulz, and P. Lievens, Phys. Rev. A 45, 4675 (1992).

[21] D. J. Berkeland, J. D. Miller, J. C. Bergquist, W. M. Itano, and D. J. Wineland, J. Appl. Phys. 83, 5025 (1998).

[22] W. Demtröder, Laser Spectroscopy (Springer-Verlag, Berlin, 1982).

[23] G. Smith, J. Phys. B 5, 2310 (1972).

[24] Atomic and Molecular Beam Methods, edited by G. Scoles (Oxford University Press, Oxford, 1988).

[25] I. Barin, Thermochemical Data of Pure Substances, 2nd ed. (VCH, Weinheim, 1993).

[26] H. W. van der Hart and C. McKenna (private communication).

[27] H. Kuhn, Atomic Spectra (Longmans, London, 1969).

[28] R. D. Cowan, The Theory of Atomic Structure and Spectra (University of California Press, Berkeley, 1981).

[29] B. B. Blinov, L. Deslauriers, P. Lee, M. J. Madsen, R. Miller, and C. Monroe, Phys. Rev. A 65, 040304 (2002).

[30] P. Bowe, L. Hornekaer, C. Brodersen, M. Drewsen, J. S. Hangst, and J. P. Schiffer, Phys. Rev. Lett. 82, 2071 (1999).

[31] I. Siemers, M. Schubert, R. Blatt, W. Neuhauser, and P. E. Toschek, Europhys. Lett. 18, 139 (1992).

[32] M. A. Rowe et al., Quantum Inf. Comput. 2, 257 (2002).

[33] G. Savard and G. Werth, Annu. Rev. Nucl. Part. Sci. 50, 119 (2000).

[34] J. I. Cirac and P. Zoller, Nature (London) 404, 579 (2000).

[35] D. Kielpinski, C. Monroe, and D. J. Wineland, Nature (London) 417, 709 (2002). 